\title{
The basic characteristics of EUV post-eruptive arcades and their role as tracers of coronal mass ejection source regions ${ }^{\star}$
}

\author{
D. Tripathi, V. Bothmer, and H. Cremades
}

\author{
Max-Planck-Institut für Aeronomie 37171 Katlenburg-Lindau, Germany \\ e-mail: [bothmer;cremades]@linmpi.mpg.de
}

Received 5 December 2003 / Accepted 5 April 2004

\begin{abstract}
The Extreme ultraviolet Imaging Telescope (EIT) on board the Solar and Heliospheric Observatory (SOHO) spacecraft provides unique observations of dynamic processes in the low corona. The EIT $195 \AA$ data taken from 1997 to the end of 2002 were investigated to study the basic physical properties of post-eruptive arcades (PEAs) and their relationship with coronal mass ejections (CMEs) as detected by SOHO/LASCO (Large Angle Spectrometric Coronagraph). Over the investigated time period, 236 PEA events have been identified in total. For each PEA, its EUV lifetime as derived from the emission time at $195 \AA$, its heliographic position and length, and its corresponding photospheric source region inferred from SOHO/MDI (Michelson Doppler Imager) data has been studied, as well as the variation of these parameters over the investigated phase of solar cycle 23. An almost one to one correspondence is found between EUV PEAs and white-light CMEs. Based on this finding, PEAs can be considered as reliable tracers of CME events even without simultaneous coronagraph observations. A detailed comparison of the white-light, soft X-ray and EUV observation for some of the events shows, that PEAs form in the aftermath of CMEs likely in the course of the magnetic restructurings taking place at the coronal source sites. The average EUV emission life-time for the selected events ranged from 2 to $20 \mathrm{~h}$, with an average of $7 \mathrm{~h}$. The heliographic length of the PEAs was in the range of 2 to 40 degrees, with an average of 15 degrees. The length increased by a factor of 3 to 4 in the latitude range of 20 to 40 degrees in the northern and southern hemispheres, with longer PEAs being observed preferentially at higher latitudes. The PEAs were located mainly in the activity belts in both hemispheres, with the southern hemispheric ones being shifted by about 15 degree in latitude further away from the solar equator during 1997-2002. The decrease in latitude of the PEA positions was 10 to 15 degrees in the northern and southern hemispheres over this period. The axes of the PEAs were overlying magnetic polarity inversion lines when traced back to the MDI synoptic charts of the photospheric field. The magnetic polarities on both sides of the inversion lines agreed with the dominant magnetic pattern expected in cycle 23, i.e. being preferentially positive to the West of the PEA axes in the North and negative in the South. One third (31\%) of the PEA events showed reversed polarities. The origin of PEAs is found not just in single bipolar regions (BPRs), but also in between pairs of neighboring BPRs.
\end{abstract}

Key words. Sun: corona - Sun: coronal mass ejections (CMEs) - Sun: flares - Sun: filaments - Sun: photosphere Sun: solar-terrestrial relations

\section{Introduction}

Coronal mass ejections (CMEs) are dynamic events in which plasma with closed magnetic field structure is ejected out of the solar atmosphere. They disrupt the flow of the solar wind in the heliosphere and can cause major geomagnetic effects (e.g., Gosling et al. 1974; Zhang et al. 2003; Bothmer \& Schwenn 1995). The origin of CMEs and their evolution in interplanetary space are not well understood. The LASCO (Large Angle Spectrometric Coronagraph) coronagraph on board the SOHO (Solar Heliospheric Observatory) spacecraft has provided

Send offprint requests to: D. Tripathi,

e-mail: tripathi@linmpi.mpg.de

* Table 1 is available in electronic form at the CDS via anonymous ftp to cdsarc.u-strasbg.fr $(130.79 .128 .5)$ or via http://cdsweb.u-strasbg.fr/cgi-bin/qcat?]/A+A/422/337 unprecedented observations of CMEs since launch in 1995 (e.g., St. Cyr et al. 2000; Howard et al. 1997).

It is a challenge to better understand the source regions of CMEs in the low corona and photosphere. Unfortunately, the regions underlying CMEs are best observed on the visible disk, whereas CMEs are best observed at the limb. Those CMEs originating around disk center are called halos (Howard et al. 1982). They appear unstructured because they propagate mainly parallel to the line-of-sight so that their real physical parameters are difficult to determine. Front-side halo CMEs are of strong importance in terms of space weather (e.g., Webb 2000; Bothmer 1999). Detailed studies of the source regions of frontside halo CMEs will provide important information to understand the solar causes of CMEs and also to help forecast their possible arrivals at Earth's orbit. 


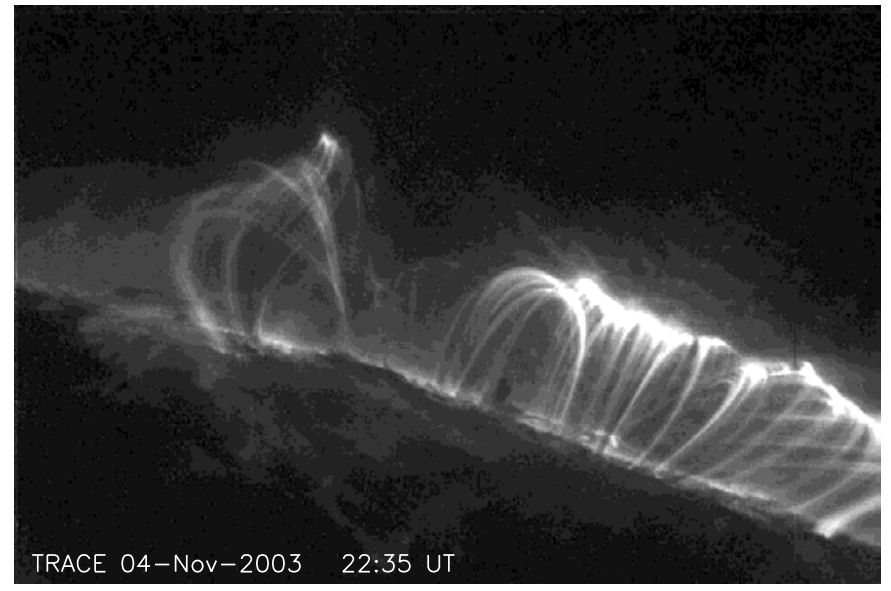

Fig. 1. A post-eruptive arcade imaged by TRACE at $195 \AA$ on 04-Nov.-2003 at 22:35 UT.

In order to identify the source regions of CMEs, erupting prominences (disappearing filaments) represent probably one of the best solar activity phenomena (e.g., Webb \& Hundhausen 1987), but for CMEs originating from active regions, often no associated disappearing filament is observed (Subramanian \& Dere 2001). Contrary to the cooler prominence material seen in absorption, brightenings of S- or reverse $\mathrm{S}$-shaped hot $\left(T 2.0 \times 10^{6} \mathrm{~K}\right)$ loop structures, called sigmoids, and developments of X-ray arcade-like loop systems were also found to be associated with the occurrences of CMEs (e.g., Rust \& Webb 1977; Svestka et al. 1998; Hudson et al. 1998). The post-eruptive arcades (PEAs), often also called post-flare loops, are also visible at EUV wavelengths (e.g., Sterling et al. 2000). A recent example for such a posteruptive arcade imaged by TRACE (Transition Region and Coronal Explorer, http://vestige.lmsal.com/TRACE/ POD/TRACEpod.html) on November 4, 2003 at 22:35 UT, which was preceded by a very fast $\left(V>2000 \mathrm{~km} \mathrm{~s}^{-1}\right) \mathrm{CME}$ and an extremely intense X-ray flare (class X28), is shown in Fig. 1. This PEA had formed at the solar limb an example of a disk PEA is shown in Fig. 2. Kopp \& Pneuman (1976) have interpreted the formation of the loop systems as a consequence of magnetic reconnection processes in the course of solar eruptions. Sometimes two ribbon flares demark the footpoint locations and developments of these loop systems.

Whereas TRACE provides detailed views of PEAs in individual events, SOHO provides a unique data set of full disk observations for solar cycle 23. Complementary to studies of smaller sets of individual events (e.g., Hudson et al. 1998; Sterling et al. 2000), the main scope of this paper is to explore the unique set of SOHO/EIT (Extreme ultraviolet Imaging Telescope) observations in order provide the first detailed statistical analysis of the general observational characteristics of EUV PEAs and their relationship to white-light CMEs detected by LASCO.

\section{Data set and event selection}

SOHO/EIT $195 \AA$ daily mpeg-movies taken from 1997 until the end of 2002 were inspected to identify PEAs on the visible solar disk together with EIT movies of higher spatial and time resolution as provided in the mvi-format $(512 \times 512$ pixels $)$. These movies commonly have a temporal resolution of $12 \mathrm{~min}$. The instrumental characteristics of EIT have been described in detail by Delaboudinière et al. (1995). The photon emission detected by EIT at $195 \AA$ is due to Fe XII ions formed at temperatures around $1.4 \times 10^{6} \mathrm{~K}$ (Dere et al. 2000). From 1996 until March 1997, the cadence of EIT data was limited due to telemetry restrictions (Subramanian \& Dere 2001).

EUV PEAs have been identified in the EIT movies by the appearance of transient brightenings of large-scale loop systems over periods of several hours. An event was selected for further study if it appeared as a clearly discernable feature on the solar disk visible over its full spatial extent (see e.g., Fig. 2). Note that this criterion excludes limb events. From the inspection of the EIT $195 \AA$ data, 236 PEA events were identified during 1997-2002 (see Table 1). The number of PEAs in 1998 was strongly decreased in the second half of the year due to the SOHO recovery phase after its preliminary loss in June. For each PEA, its spatial extent was located in the EIT images at times around its maximum brightness at $195 \AA$. The heliographic coordinates of the arcade's extreme ends were determined in latitude and longitude listed as $b_{1}, l_{1}, b_{2}$ and $l_{2}$ in Table 1 , with $\left(b_{1}, l_{1}\right)$ and $\left(b_{2}, l_{2}\right)$ denoting the PEA's end points to the West and East respectively. denoting the arcade's endpoint to the East (the values provided in Table 1, maybe compared with the top panel of Fig. 2 in Sect. 3). For PEAs that can be considered as fairly linear features, to first order $\left(b_{1}, l_{1}\right),\left(b_{2}, l_{2}\right)$ represent the endpoints of the mid axis of the arcade. The calculation of the length $L$ of each arcades based on these values is described in detail in Sect. 3. The approximated lifetime of each PEA was determined from its presence as a discernible feature in the EIT $195 \AA$ images with a common time-cadence of $12 \mathrm{~min}$.

The correlation of PEAs with CMEs was studied using data from the SOHO/LASCO coronagraphs. A detailed description of LASCO was given by Brueckner et al. (1995). The LASCO/C2 coronagraph images and movies provide whitelight observations over a field of view (FOV) from 1.5 to 6.0 solar radii with a corresponding pixel size of 11.4 arcsec and a time resolution of $20 \mathrm{~min}$. The spatial correlation of PEAs with CMEs was investigated by comparing the CME's position angle (PA) as provided by the CME catalogue (http://cdaw.gsfc.nasa.gov/CMElist/, Yashiro et al. 2002) with the solar positions of the related PEAs (compare with Fig. 2 in Sect. 3). The PA is measured positive in the counter-clockwise direction, starting with zero degrees in the solar North. In order to prove the association of a given PEA with a CME detected by LASCO/C2, the temporal correlation of both phenomena was further investigated by comparing the observation time of the PEA with the height-time (hereafter h-t) diagram of the CME as taken from the CME catalogue including both LASCO C2 and C3 (FOV 1.5-6.0 $R_{\odot}$ \& 4.0-30 $R_{\odot}$ ) measurements. The time of the first detection of each related CME in C2 is specified in the last column of Table 1. From the h-t diagrams the approximate CME onset times were estimated together with their outward evolution and compared with the approximate onsets of the disturbances 


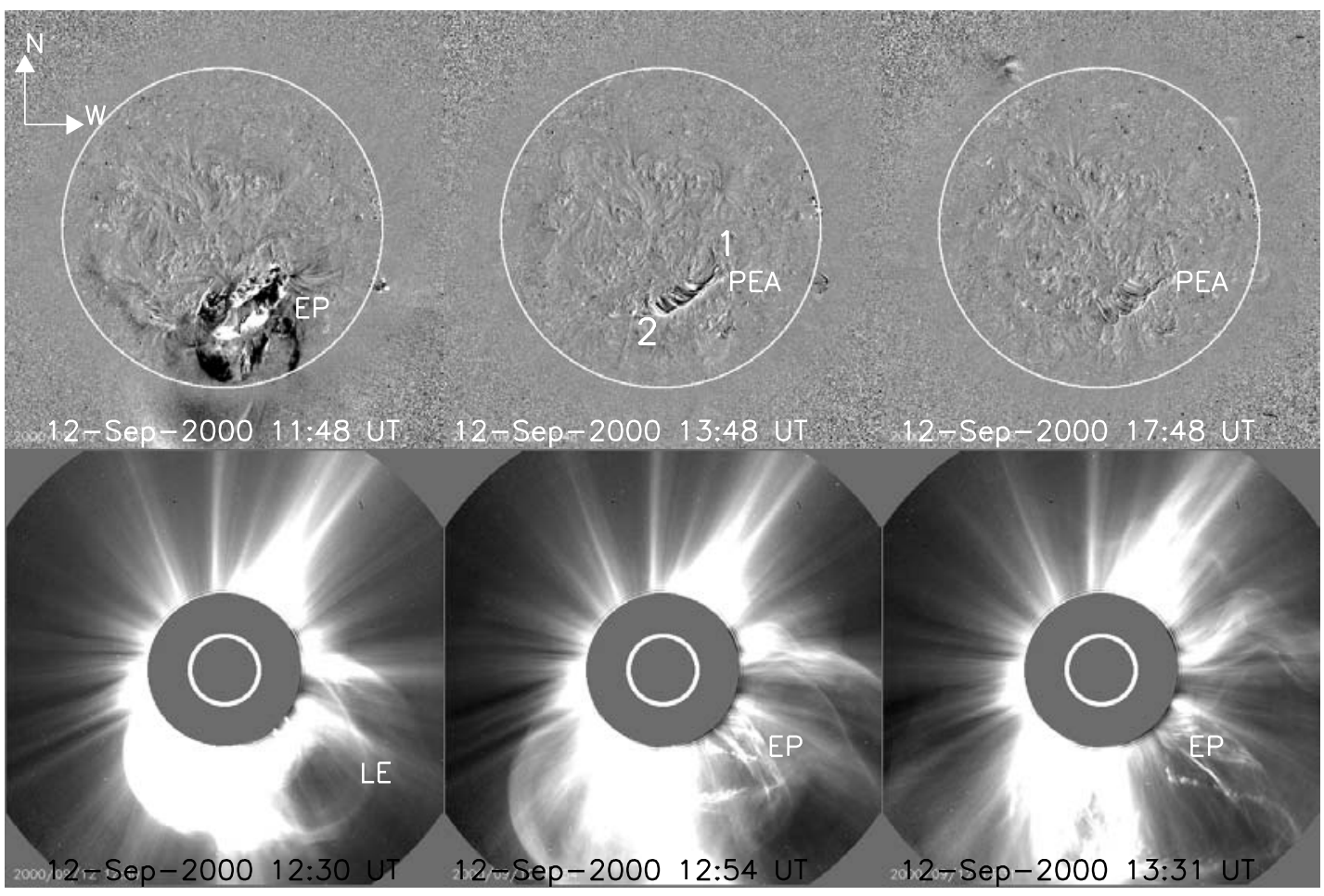

Fig. 2. Top panel: running difference images taken by EIT $195 \AA$ on 12-Sep.-2000. The first two images show the erupting prominence (EP) event and consequent post-eruptive arcade (PEA) formation in the southern hemisphere near CM. Points 1 and 2 represent the start and end points of the PEA. The last figure reveals the dimming of the arcade. Bottom panel: LASCO/C2 images showing the evolution of the associated CME in white-light. LE denotes the leading edge of the CME and EP the erupting prominence. In all figures, north is up and west is towards the right. The speed of the CME in the plane of the sky was $1550 \mathrm{~km} \mathrm{~s}^{-1}$ at PA 220.

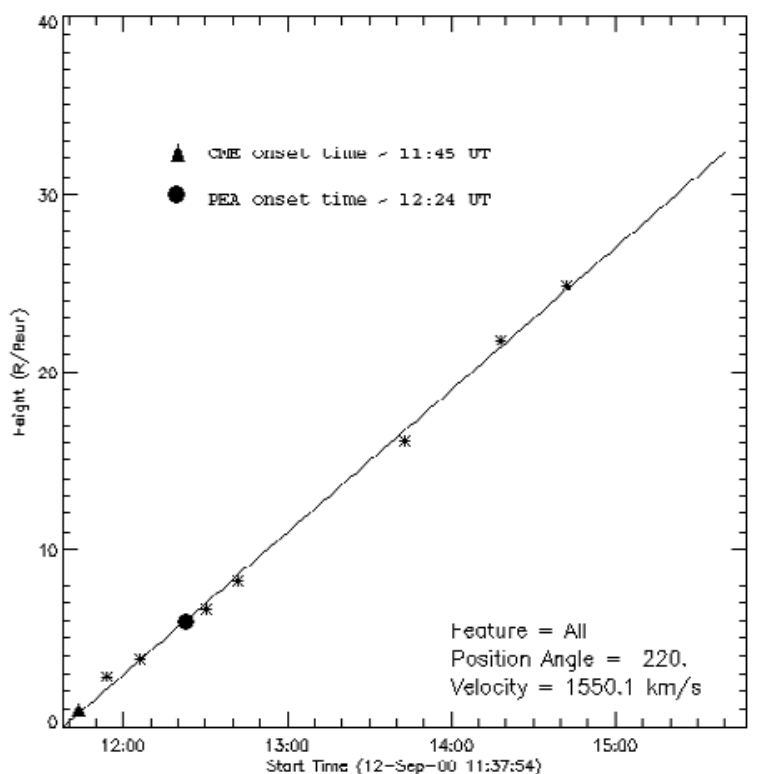

Fig. 3. LASCO $\mathrm{C} 2 / \mathrm{C} 3$ h-t diagram for the $\mathrm{CME}$ detected on 12-Sep.-2000, around 12:30 UT. The solid triangle marks the estimated CME onset time at 1 solar radii and the solid circle represents the identified onset time of the PEA based on EIT $195 \AA$ images. The h-t diagram was taken from the CME catalogue at http://cdaw.gsfc.nasa.gov/CME_list/.

in the low corona and the PEA developments (see Fig. 3 in Sect. 3).
In order to study the underlying photospheric field signature of the PEAs, magnetograms from the SOHO/MDI (Michelson Doppler Imager, see Scherrer et al. 1995) instrument were used. MDI measures the line-of-sight, i.e. the longitudinal component of the magnetic field with a resolution of $2 \operatorname{arcsec}$ via the Ni I (6768 $\AA$ ) line. In order to compare the EIT PEA events with other solar activity features, such as disappearing filament/prominence eruptions, X-ray flares or soft X-ray brightenings, data from the Paris/Meudon (http://bass2000.obspm.fr/ home.php), and Big Bear (ftp://ftp.bbso.njit.edu/ pub/archive/) observatories, the GOES satellite (ftp:// ftp.ngdc.noaa.gov/STP/SOLARDATA/SOLAR_FLARES/ XRAY_FLARES/) and the Soft X-ray telescope (SXT) on board Yohkoh (http:// cdaw.gsfc.nasa.gov/CME_list/ daily_mpg/; see Tsuneta et al. 1991) were consulted.

\section{Association of PEAs with CMEs}

The association with a white-light CME was investigated for every identified PEA listed in Table 1 based on LASCO/C2 observations as described in Sect. 2. Figure 2 shows an example of a PEA located near central meridian (CM) in the southern solar hemisphere and its associated white-light CME first detected in the FOV of C2 on 12 September 2000, at 12:30 UT. The detection of the CME in C2 was preceded by a filament eruption observed by EIT at around 11:48 UT, becoming visible in $\mathrm{C} 2$ at 12:54 UT. The estimated onset time of the CME 
Table 2. The columns from left to right are: Date of observation; start-, end of rising- and peak-time of GOES X-ray flares; flare locations on the solar disk in heliographic coordinates; times of coronal brightenings observed by Yohkoh/SXT, onset times of PEA observed by SOHO/EIT at $195 \AA$; estimated onset times of the associated CMEs based on the investigation of h-t diagrams.

\begin{tabular}{lcccccc}
\hline \hline Date & $\begin{array}{c}\text { GOES } \\
\text { X-ray } \\
(\text { UT })\end{array}$ & $\begin{array}{c}\text { Flare } \\
\text { location } \\
(\text { deg })\end{array}$ & $\begin{array}{c}\text { Flare } \\
\text { class }\end{array}$ & CB & $\begin{array}{c}\text { PEA } \\
\text { onset } \\
(\text { UT })\end{array}$ & $\begin{array}{c}\text { CME } \\
\text { onset } \\
(\text { UT })\end{array}$ \\
\hline 9-Feb.-00 & 19:10-19:18; 19:40; 20:00 & S17 W40 & C74 & $(19: 43)$ & $20: 12$ & $19: 26$ \\
17-Feb.-00 & 20:10-20:15; 20:30; 20:32 & S29 E07 & M13 & $(20: 23)$ & $20: 36$ & $20: 19$ \\
2-Jun.-00 & $08: 50-09: 00 ; 09: 30 ; 09: 36$ & N10 E23 & C24 & $(09: 22)$ & $09: 36$ & $09: 12$ \\
6-Jul.-00 & 12:15-12:20; 12:35; 12:36 & N18 E25 & C43 & $(13: 15)$ & $13: 25$ & $12: 30$ \\
\hline
\end{tabular}

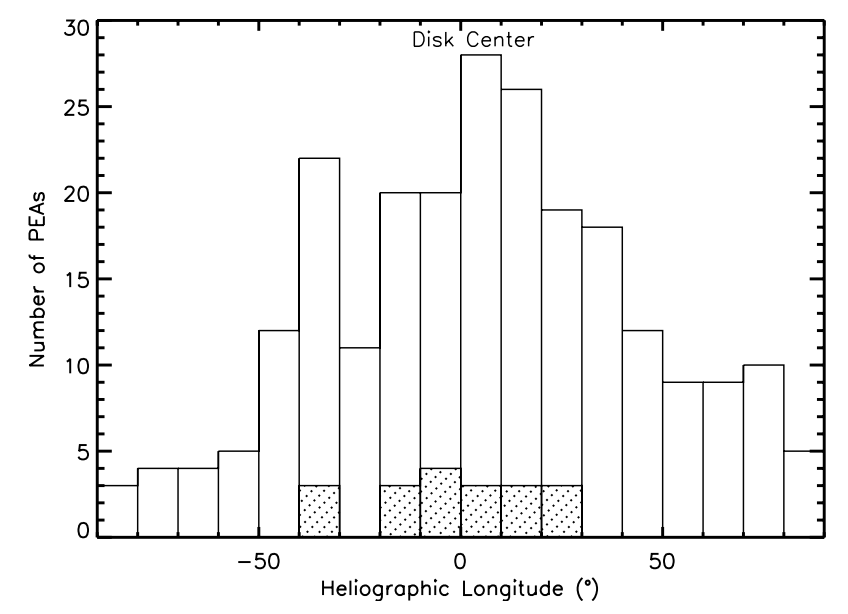

Fig. 4. Distribution of PEAs in heliographic longitude in bins of 10 degrees as identified in EIT $195 \AA$ Amages from 1997 to 2002. The portions of the bars represented as spotted areas represent those PEAs for which no white-light CME had been detected by LASCO/C2.

was 11:45 UT according to the h-t diagram (see Fig. 3), which in this case is in good agreement with the onset time of the prominence eruption imaged by EIT. However, the validity of the estimated CME onset time based on the extrapolation of the h-t diagram can be misleading, e.g., for CMEs that were slowly accelerated in its initiation phase or in case of halo CMEs. The identified onset time of the corresponding PEA at $195 \AA$ in the EIT images was 12:24 UT, showing its maximum development around 13:48 UT and disappearing at about 17:48 UT. The EUV PEA formed about 39 min after the eruption of the prominence and CME lift-off, and was still lasting when the CME had already reached a height of more than $40 R_{\odot}$. The timing of the individual phenomena is summarized in Fig. 3.

A remarkable feature of Fig. 2 is the apparent similarity between the shape of the CME core and structure of erupting prominence and PEA system. As can be inferred from the bottom panel of Fig. 2, the CME resembles a large-scale curved cylindrical flux tube that has originated from a source site bounded by the PEA's start and end points which are likely to be considered as the two legs of the CME.

The systematic investigation of the association of the entire set of PEA events listed in Table 1, with white-light CMEs detected by LASCO in close space and time relationship, showed that $210(92 \%)$ out of 236 events identified from 1997 until the end of 2002 had clear CME associations. In seven events there were no LASCO data taken, so that only 19 (8\%) PEAs were found to be lacking an associated CME.

Figure 4 shows the distribution of all identified PEAs in heliographic longitude presented in bins of ten degrees. As expected, the distribution peaks near CM, where PEA events can best be observed over its full extent. The few number of PEAs without associated LASCO/C2 CMEs were all observed around disk center in the range $40^{\circ}$ East to $30^{\circ}$ West.

Taking into account, that front-side halo CMEs originating from near disk center are often hard to detect because of the dependence of the efficiency of Thomson scattered light on the viewing angle of the CME with respect to the line of sight (e.g., Plunkett et al. 1998; Brueckner et al. 1998), it seems reasonable to assume that the small number of PEAs without CME association may have been caused by sensitivity limitations of LASCO. It thus seems plausible to assume that PEAs are definite indicators of CMEs that originated from the corresponding regions of the visible disk.

Disappearing filaments (prominence eruptions) are considered as one of the most unique signatures of CMEs (e.g., Bothmer \& Schwenn 1994). As an example for this connection, Fig. 5 shows that the front-side halo CME on February 17, 2000, that originated from the SE part of the Sun near CM, was accompanied by a filament disappearance in $\mathrm{H} \alpha$ (Fig. 5, middle and right images in top panel). Figure 6 shows a multi-wavelength view of the CME's source region based on SOHO/EIT and MDI, Yohkoh/SXT, and ground-based observations from Paris/Meudon. The filament closely followed the orientation of the neutral line separating regions of opposite magnetic polarity as inferred from the SOHO/MDI data. Bright loops in form of an S-shaped sigmoid, prominent in the Sun's southern hemisphere (e.g., Canfield et al. 1999), are often visible shortly before the eruption of a CME, visible here at around 20:00 UT on February 17 (Fig. 6, second panel from top, first image). A strong brightening of the sigmoidal system occurred near the CME's liftoff time at around 20:23 UT (see Yohkoh/SXT images in Fig. 6), followed by the onset of the EUV arcade around 21:36 UT. The X-ray flare observed by GOES 8 (available at http : //www . lmsal . com/SXT/plot_goes .html) started between 20:10 and 20:15 UT (see Table 2), increasing in intensity and reaching its peak value near 20:32 UT. The onset of the PEA seen by EIT follows in time the peak of the X-ray flare. The CME onset time corresponds to the rising phase of the X-ray flare, coinciding with its acceleration phase, in 


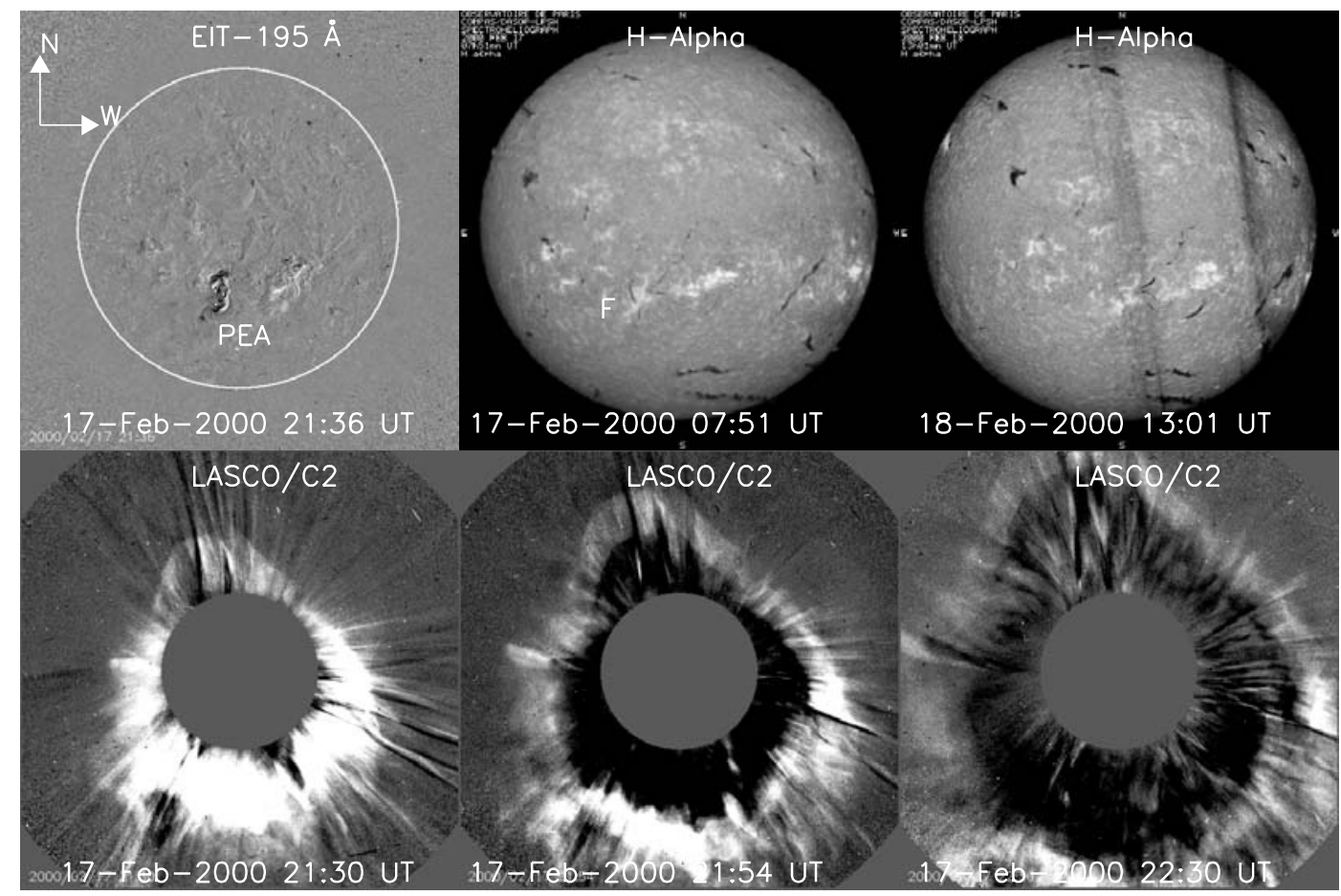

Fig. 5. Top panel: the first image shows a running difference image taken by EIT at $195 \AA$ A on 17-Feb.-2000 displaying the PEA that had formed in the southern hemisphere. The second and third images show that a filament (F) disappeared from this solar region based on H $\alpha$ observations taken on 17 and 18 February 2000 (courtesy Paris/Meudon Observatory). Bottom panel: the associated halo CME on February 17, 2000 as detected by LASCO/C2 around 21:30 UT. The speed of the CME was $600 \mathrm{~km} \mathrm{~s}^{-1}$ at PA 196.

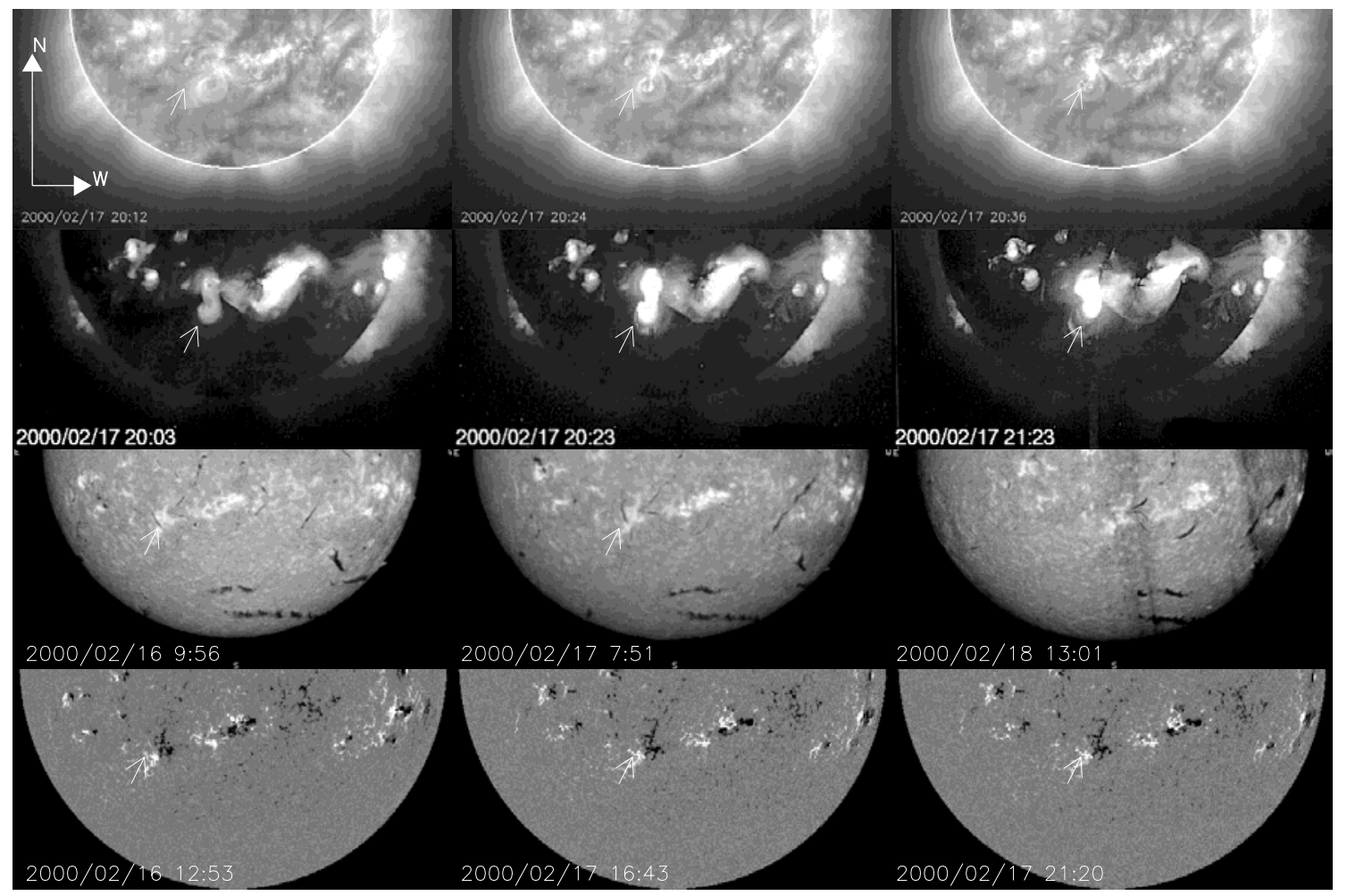

Fig. 6. Multi-wavelengths observations for the source region of the 17-Feb.-2000 PEA event around 21:30 UT. Top to bottom: First panel: EIT $195 \AA$ images taken at 20:12 UT, 20:24 UT and 20:36 UT on February 17, 2000 showing the pre-eruption configuration of the halo CME detected later by LASCO and the initial phase of the arcade formation. Second panel: Yohkoh/SXT images taken at 20:03 UT, 20:23 UT and 21:23 UT on the same day showing the brightening of an S-shaped sigmoid in the S-hemisphere in the CME source region. Third panel: H $\alpha$ observations taken on 16, 17 and 18 February 2000 showing that a filament had disappeared from the corresponding source region. Fourth panel: MDI magnetograms taken at 12:53 UT on February 16, 16:43 UT and 21:20 UT on February 17, showing the photospheric magnetic field structure of the CME's source region. 


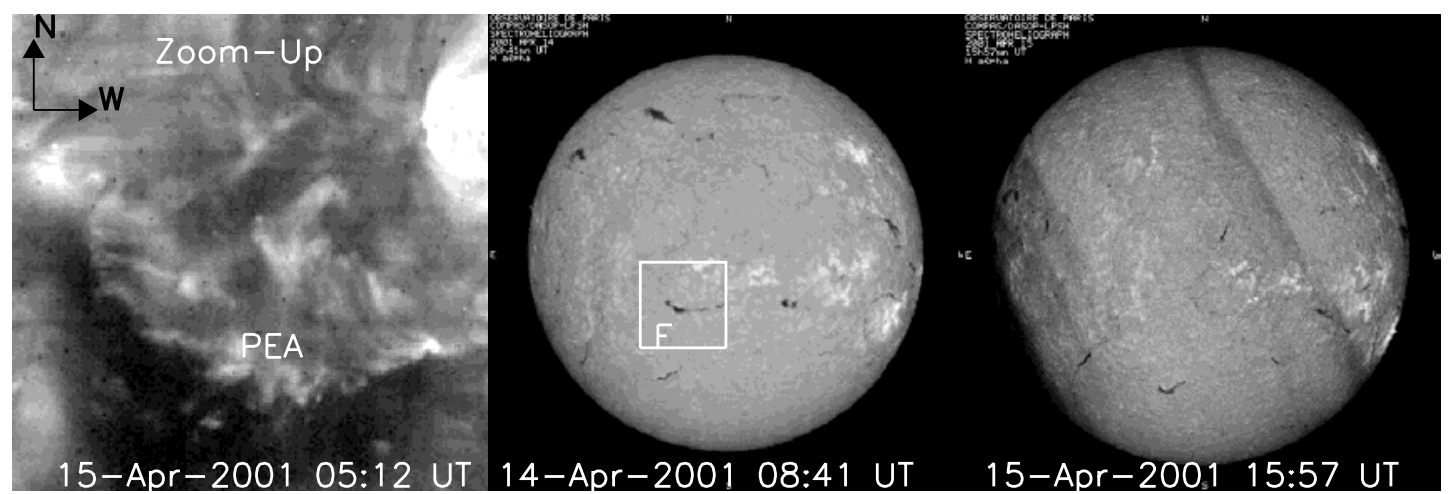

Fig. 7. Left to right: zoomed view of the post-eruptive arcade (PEA) observed by EIT at $195 \AA$ on 15-Apr.-2001 at 05:12 UT followed by two $\mathrm{H}$ - alpha images taken on 14 and 15 April showing the position of the corresponding disappearing filament $(\mathrm{F})$ on the solar disk.
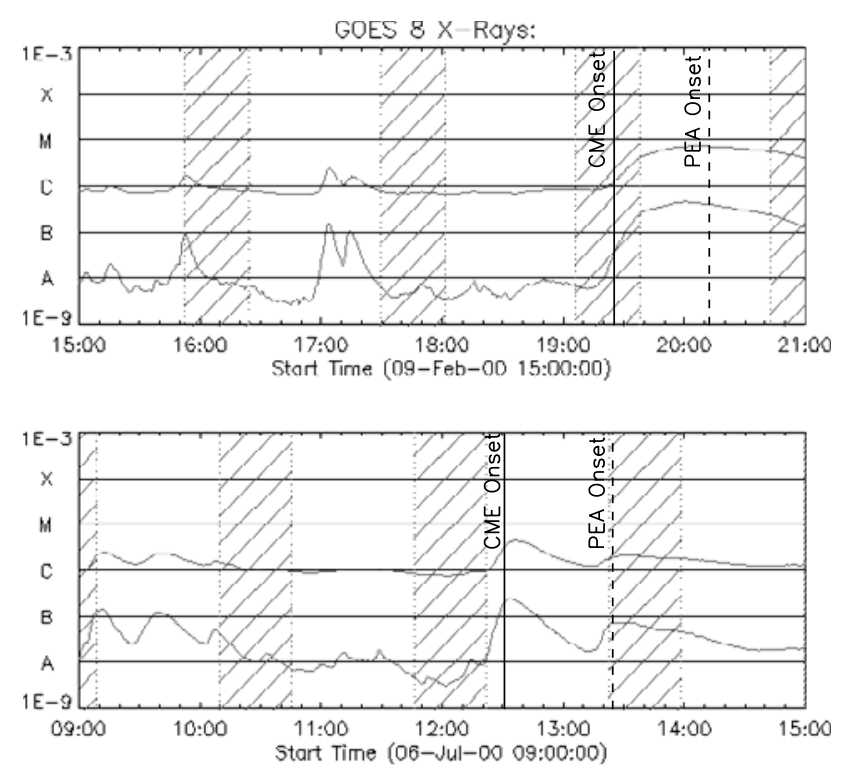

Fig. 8. GOES 8 X-ray plots for the CME and PEA events on February 9, 2000 (top) and 6 July, 2000 (bottom). The approximate onset times of the CMEs and PEAs are labeled.

agreement with the findings of Zhang et al. (2001). Assuming that the peak intensity of the flare indicates the CME's lift-off from the Sun, the PEA may be interpreted as a consequence of magnetic reconnection processes that were initiated underneath the rising CME as suggested by Kopp \& Pneuman (1976).

For three more PEA events, the flare position, as provided by the GOES catalogue (ftp://ftp.ngdc.noaa.gov/ STP/SOLAR_DATA/SOLAR_FLARES/XRAY_FLARES), was located not further away from the PEA location than $\pm 5^{\circ}$ in heliographic latitude and longitude and which were observed within a time interval of three hours prior to the onset of the PEA, the chronological evolution of the coronal features seen by EIT $195 \AA$ and SXT were compared with the GOES 8 X-ray flare timings (Table 2). The time evolution in the events is similar: A rising phase in soft X-ray intensity during the acceleration phase of the CME, its consequent lift-off and propagation phase, followed some minutes later by the peak in X-ray intensity, finally followed by the formation of the PEA underneath it. Figure 8 shows the time evolution of the GOES 8 X-ray flare for the PEAs and CMEs observed on

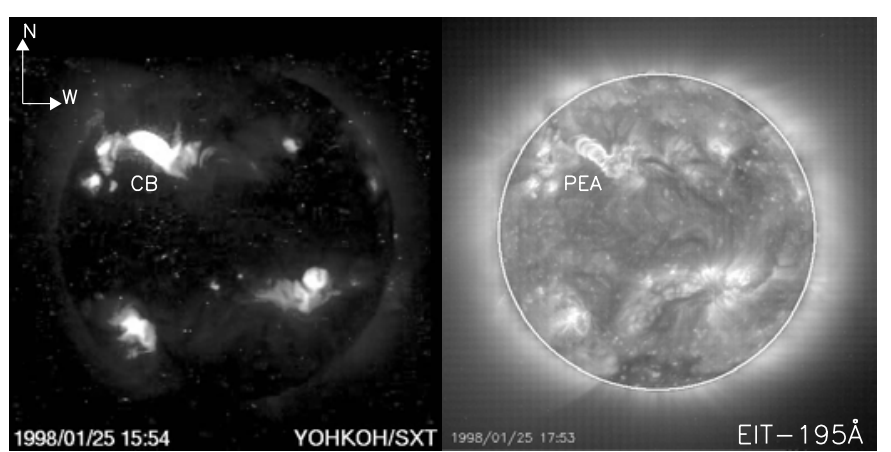

Fig. 9. YOHKOH/SXT image taken on 25-Jan.-1998 at 15:54 UT showing the coronal brightening (CB) that was detected on the NE part of the solar disk and the corresponding post-eruptive arcade (PEA) observed by EIT at $195 \AA$ at 17:53 UT.

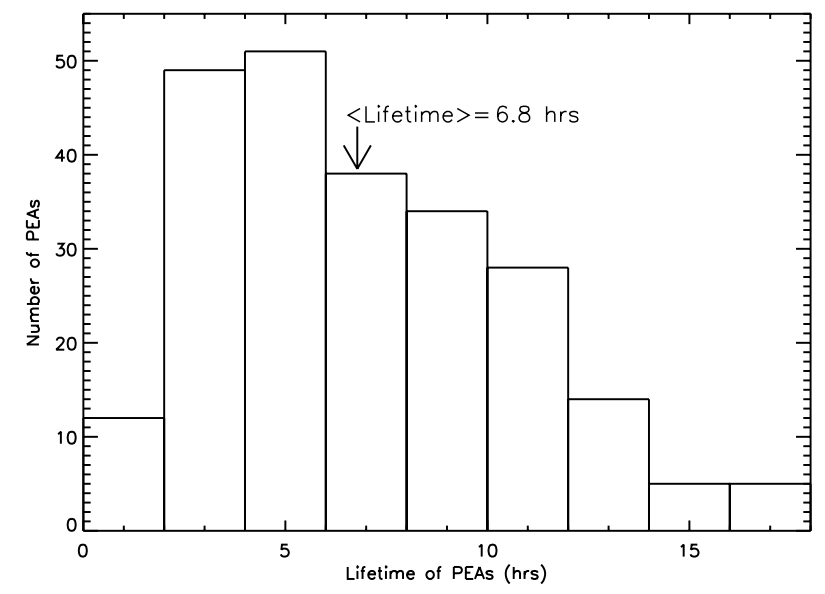

Fig. 10. Frequency distribution of the lifetimes of the identified PEAs during 1997-2002 based on SOHO/EIT $195 \AA$ observations in bins of $2 \mathrm{~h}$. The average value for the lifetime was $7 \mathrm{~h}$.

February 9 and July 6, 2000. Whereas on July 6, 2000, the PEA causes a second increase of the X-ray intensities, it can not be distinguished as a separate feature in the GOES 8 X-rays on February 17, 2000. The coronal brightenings observed by SXT preceded the onset of the EUV PEAs by about 5 to 30 min (see Table 2), but due to the irregular cadence of the SXT observations, no detailed statistics could be performed. Figure 9 shows an example of a transient coronal brightening 


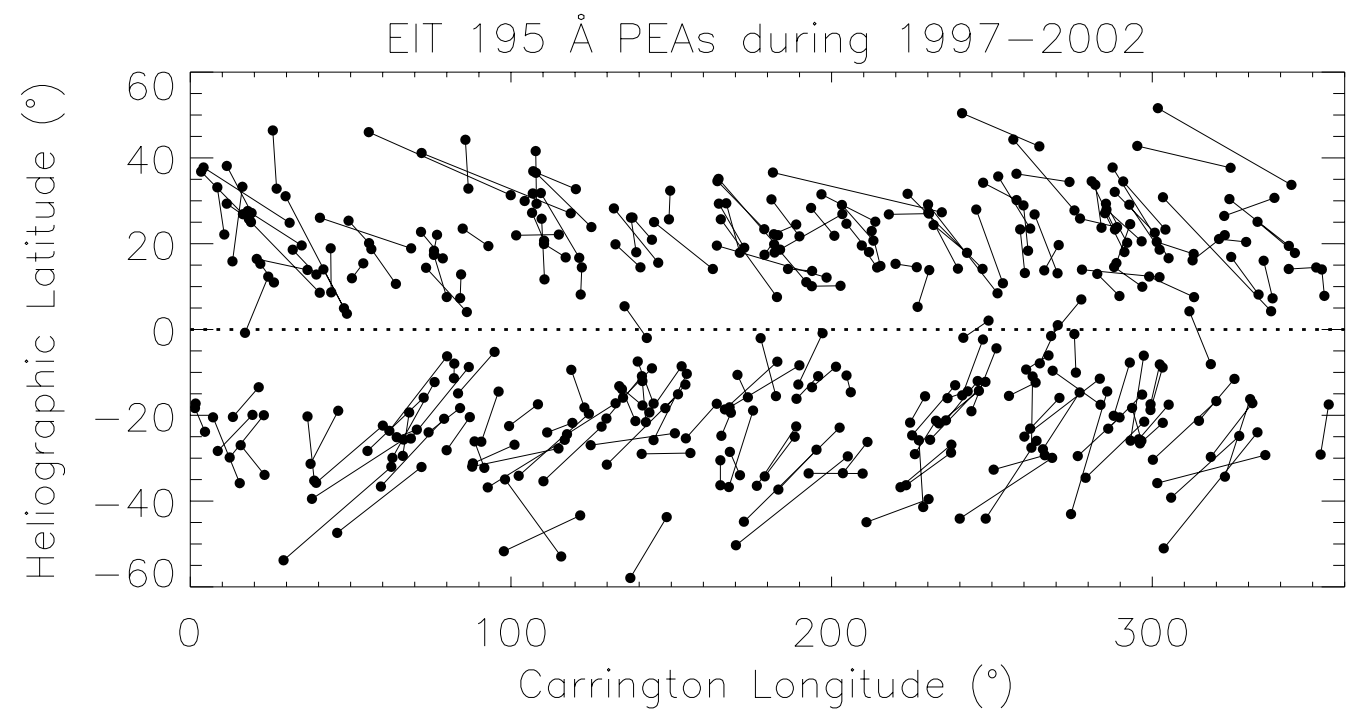

Fig. 11. Carrington synoptic map representing the positions of all identified PEAs during 1997-2002 based on the analysis of SOHO/EIT $195 \AA$ observations.

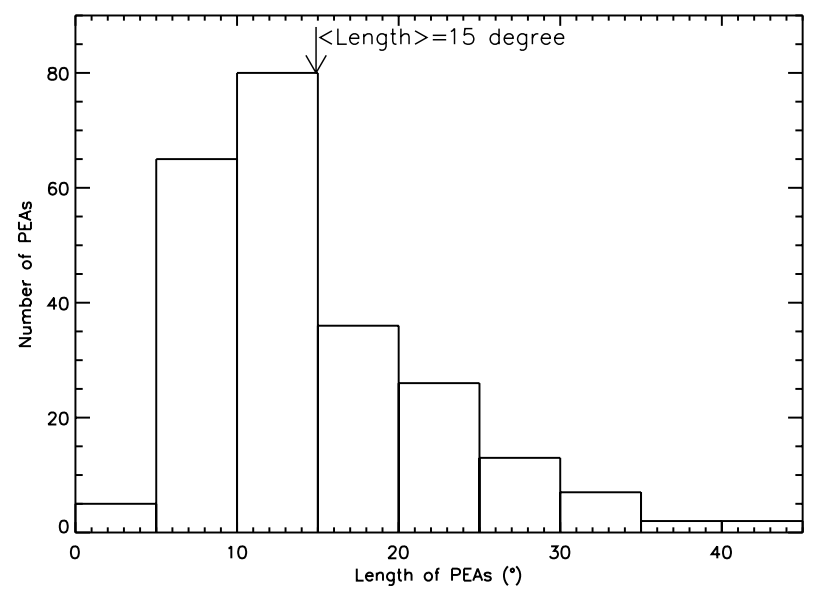

Fig. 12. Frequency distribution showing the heliographic lengths of PEAs identified from SOHO/EIT 195 A images during 1997-2002 in bins of $5^{\circ}$. The average length of the identified PEAs was $15^{\circ}$.

detected by SXT on 25-Jan.-1998 and post-eruptive arcade seen by EIT.

A PEA event associated with a disappearing filament, but without corresponding LASCO white-light CME is shown in Fig. 7. For better visibility, only a portion of the full disk EIT image, comprising the full spatial extent of the PEA is displayed. The $\mathrm{H} \alpha$ and EIT features are basically the same as observed for the event on 17 February 2000, visualized in Figs. 5 and 6, except that a CME was not identified in the LASCO data.

If one considers $\mathrm{H} \alpha$ filament eruptions as valid CME proxies, 14 more events out of the 19 cases where no white-light CME was detected, can be clearly considered as probably CME associated. Unfortunately, in 3 cases no $\mathrm{H} \alpha$ data were available and in 2 cases the events were very complex so that the interpretation of the data was difficult (see Table 1). If one takes into account these informations, the total number of PEAs

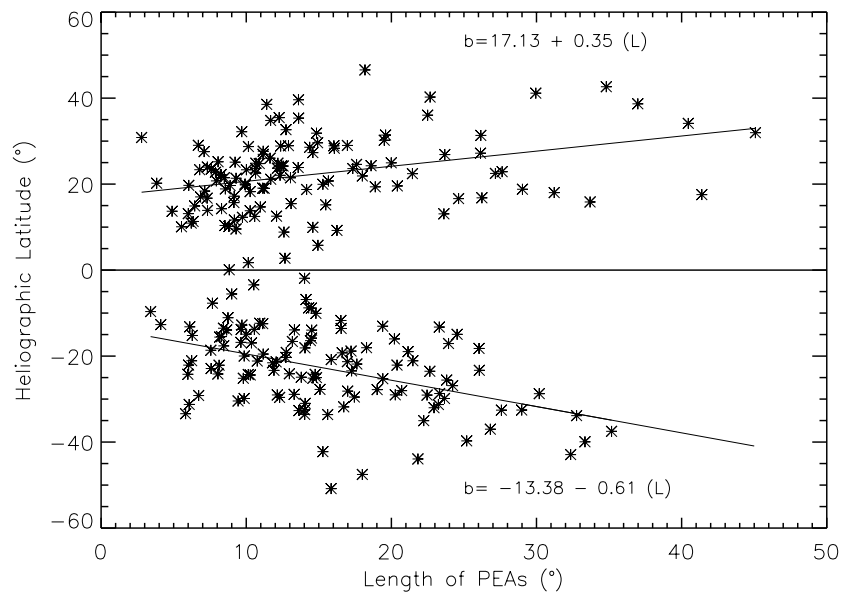

Fig. 13. Variation of PEA lengths with heliographic latitude during 1997-2002. The data points represent the latitude for the midpoint of the PEA axes. Note that no events were identified above $60^{\circ}$. The straight lines represent the fitted latitudinal trends in both solar hemispheres in the range 20 to 40 degree latitude, with: $L_{\mathrm{N}}(\mathrm{deg})=$ $2.9(b-17.1)$ and $L_{\mathrm{S}}(\mathrm{deg})=-1.6(b+13.4)$, with $b$ being the heliographic latitude of the mid point of a PEA axis. Positive values of the latitude correspond to the northern hemisphere.

associated with CME increases to $98 \%$ (224 out of 229 events) i.e. almost a one to one correspondence is found between EUV PEAs and CMEs.

\section{Basic properties of PEAs}

\subsection{EIT $195 \AA ̊$ emission lifetime}

For each of the PEA events, the time interval was determined over which the EUV arcade could be clearly distinguished in the EIT images at $195 \AA$ with a usual time resolution of $12 \mathrm{~min}$. The start time of the events was defined as the moment when 


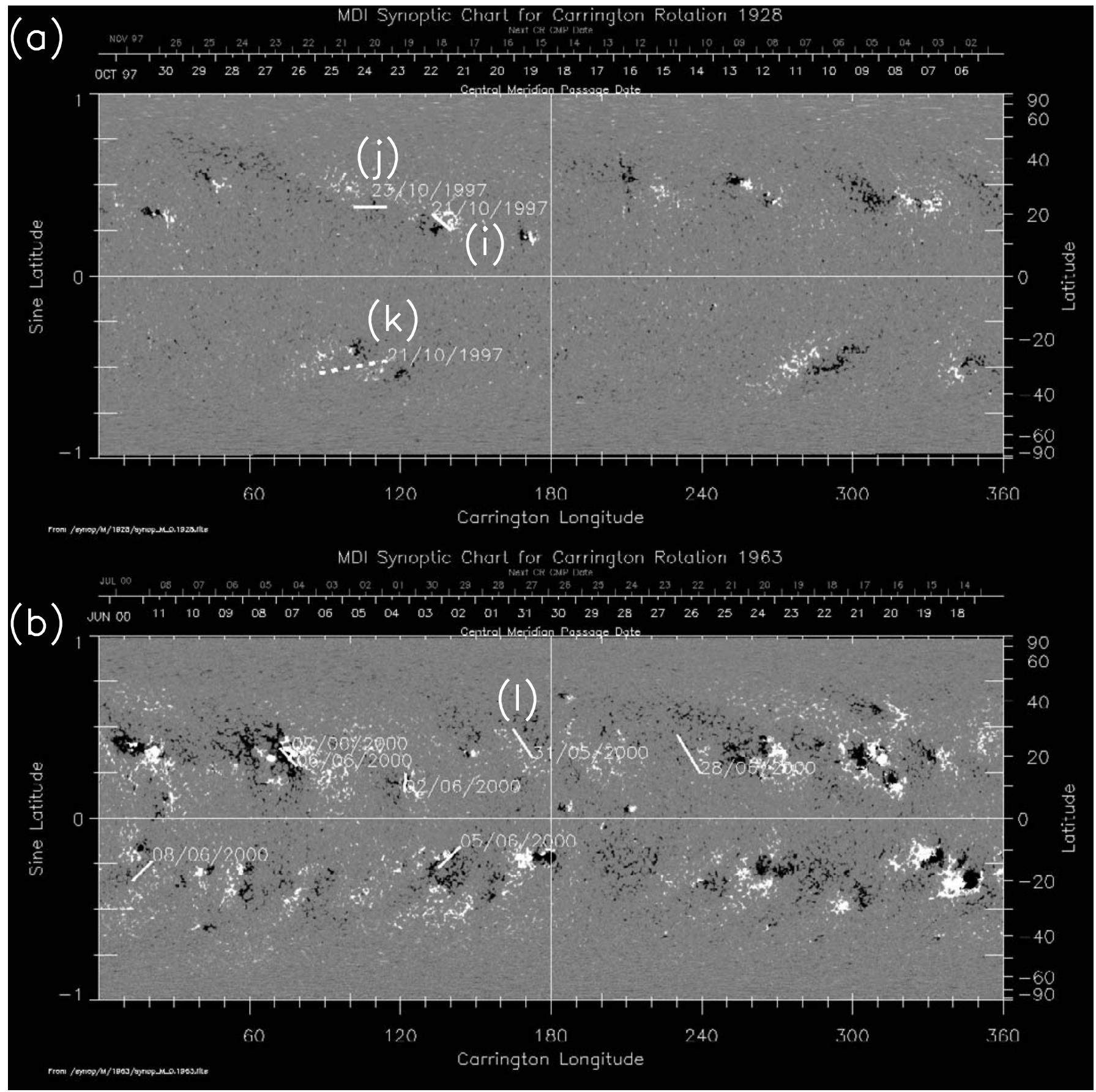

Fig. 14. SOHO/MDI Carrington synoptic charts for rotations 1928 a) in 1997 and 1963 b) in 2000 and identified source regions of nine posteruptive arcades (PEAs), marked by solid and dashed lines. In the synoptic charts, white colors represent areas of positive magnetic polarity, black colors those of negative polarity. Note that in the North the leading areas reveal positive magnetic polarities in this cycle and vice versa in the South. The solid and dashed lines represent the individual PEA axes. Case i) and j) indicates a linear shaped PEA. A PEA located in between two bipolar regions is labeled as $\mathrm{k}$ ) and a case untypical for the dominant polarity in the northern hemisphere in cycle 23 as 1 ) polarity configuration.

the large scale loop system could be identified the first time in the EIT images and the end time was defined as the time when the loops could not be distinguished anymore. All estimated lifetimes are listed in Table 1. Figure 10 shows the EIT $195 \AA$ lifetime distribution of the PEAs in bins of $2 \mathrm{~h}$. The typical lifetime of a EUV PEA ranged from 2 to $10 \mathrm{~h}$, with an average lifetime of about $7 \mathrm{~h}$.

\subsection{Heliographic position, length and variation with latitude}

For each PEA, the start and end points of the loop system's long axis were calculated as described in Sect. 2 (see Table 1). Figure 11 displays the heliographic positions of all PEAs identified during the years 1997 to 2002 in a single Carrington map. 
Figure 11 shows that most of the PEAs formed in the heliographic latitude range $\pm 40^{\circ}$ North and South and that transequatorial cases were extremely rare. No PEA was observed at latitudes above $60^{\circ}$ North or South. The orientation of the PEA axes followed Joy's law for the tilt of sunspots (Hale et al. 1919), i.e. the tilt angle of sunspots is half of the latitude, i.e. a sunspot at $60^{\circ}$ North would be expected to have a tilt of about $30^{\circ}$, as measured from East towards North. The tilt angles in sunspots are measured from the equator towards North in the northern hemisphere and vice versa in the southern hemisphere.

Based on the estimated heliographic coordinates, the length $(L)$ of each PEAs was calculated as:

$L=\arccos \left(\sin \left(b_{1}\right) \sin \left(b_{2}\right)+\cos \left(b_{1}\right) \cos \left(b_{2}\right) \cos \left(l_{1}-l_{2}\right)\right)$.

The parameters $\left(b_{1}, l_{1}\right)$ and $\left(b_{2}, l_{2}\right)$ are the heliographic coordinates of the PEA's first and second points as defined in Sect. 2.

Figure 12 shows the frequency distribution of the calculated lengths of the PEAs. The length of the PEAs varied from 2 to 40 degrees, with an average value of about $15^{\circ}$. As indicated by Fig. 11, PEAs exceeding the average length seem to be observed preferentially at higher heliographic latitudes. The length variation with heliographic latitude in both solar hemispheres is shown in Fig. 13. From a linear polynomial fit it is found, that the length $(L)$ of PEAs increases with latitude in the North as $L_{\mathrm{N}}(\mathrm{deg})=2.9(b-17.1)$ and in the South as $L_{\mathrm{S}}(\mathrm{deg})=-1.6(b+13.4)$, with $b$ being the heliographic latitude. For latitudes of 20, 30 and 40 degrees North and South this corresponds to PEA lengths of 8, 37 and 66 degrees in the North, and 11, 27 and 43 degrees in the South. Since PEAs can be considered as tracers of the source regions of CMEs, this finding may imply that the longitudinal extension of CMEs increases with heliographic latitude. The increase of the PEA length with latitude appears to be associated with the larger sizes of disappearing filaments at higher latitudes.

\section{Photospheric source regions and solar cycle dependence}

The estimated heliographic position for each PEA was used to locate its photospheric source region in the SOHO/MDI magnetogram synoptic charts taken during the corresponding CR. For simplicity, no consideration of the time differences between the arcade occurrences and the observation dates of the single magnetograms that contributed to the individual synoptic maps were taken into account here. The synoptic charts are generated from the definitive MDI magnetograms (available at http://soi.stanford.edu/magnetic/index 5.html). For this study, the charts produced from near central meridian observations were used. This implies that arcade events identified in the eastern solar hemisphere had appeared in time after the magnetograms used to construct the synoptic maps were taken, whereas western hemispheric events had preceded the magnetogram observations.

Figures 14a,b show the positions of the ten EUV arcades identified in CRs 1928 and 1963. White and black colors in the magnetograms represent positive (field lines pointing
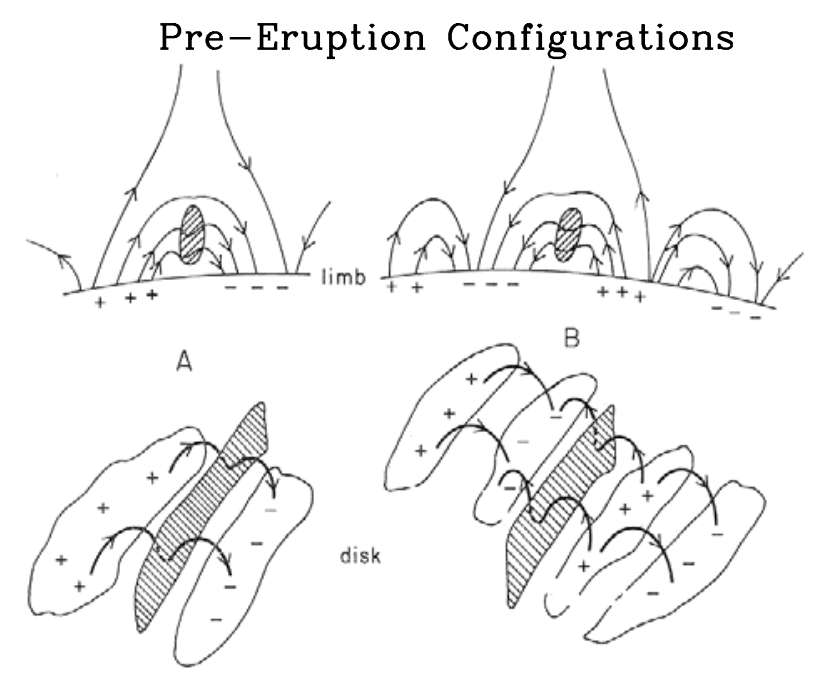

Post-Eruptive Arcades

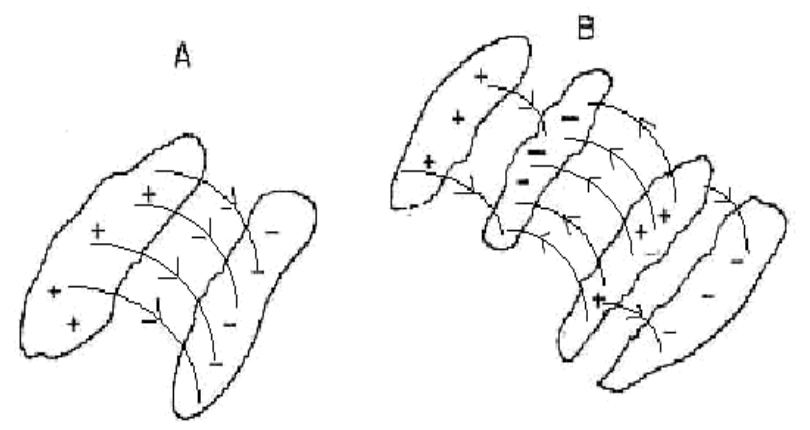

Fig. 15. Sketch showing the possible pre-eruption field configuration of PEAs forming along neutral lines/filament sites in single bipolar regions (A) and in between pairs of them (B). The pre-eruption configuration is adapted from Tandberg-Hanssen (1974).

away from the photosphere) and negative (field lines pointing towards the photosphere) magnetic polarities. The solid and dashed lines in Fig. 14 mark the calculated PEA long axes positions of the three selected PEAs of CR 1928 and the seven of CR 1963. The axes positions always matched the positions and orientations of polarity inversion lines (PILs, neutral lines) separating regions of opposite magnetic polarities in both solar hemispheres similar to the conclusion of Subramanian \& Dere (2001).

Figure 14a shows data from October 1997, i.e. in the rising phase of solar cycle 23, and Fig. 14b shows data for June 2000 , i.e. around solar activity maximum. The number of active regions and the photospheric magnetic flux is considerably higher near solar maximum. This difference is also reflected by the minor number of PEAs identified in CR 1928. The comparison of the EIT and MDI observations yielded, that PEAs can either comprise an entire PIL of a bipolar region (Fig. 14a, case i) or they just a fraction of it (Fig. 14a, case j). Besides the cases in which PEAs had formed associated with specific single BPRs, some PEAs were found to be located in between two neighboring bipolar region (see Fig. $14 \mathrm{a}(\mathrm{k})$ ). The magnetic configurations shown in Fig. 15, in analogy to the ones suggested by Tandberg-Hanssen (1974, p. 118), naturally represents the preeruption magnetic structure for both types of events. In Fig. 15, the presence of a filament is assumed in both configurations, 


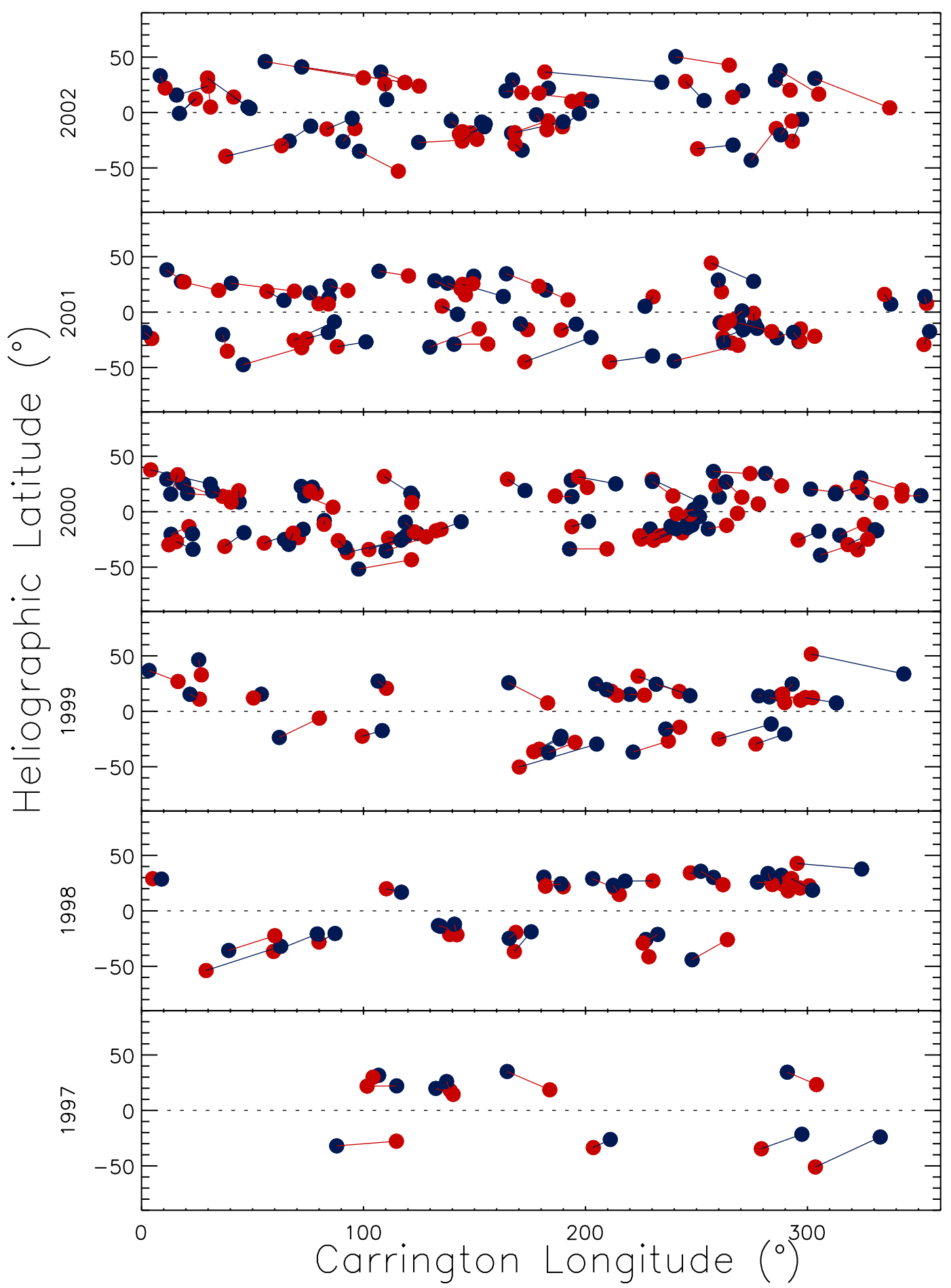

Fig. 16. Carrington synoptic charts of the heliographic positions of post-eruptive arcades identified during 1997-2002 for which the magnetic polarities of the source regions were identified from MDI synoptic charts. The colors at the equatorward ends of the PEAs denote the magnetic polarity to the West of the individual polarity inversion line, with the red color being assigned to the positive magnetic polarity and the blue color assigned to the negative one.

although it is not necessarily required. Although the magnetic polarities on both sides of the PILs were most often the expected ones for cycle 23, with leading positive polarities in the sense of solar rotation in the North and leading negative polarities in the South, some of the PEAs formed in regions of reverse polarity configuration as compared to the one dominating the given cycle (Fig. 14b(1)).

Out of the 236 PEA events listed in Table 1, in 216 cases the magnetic polarities on both sides of the arcade's axis could be uniquely identified. The remaining events occurred at latitudes above $40^{\circ}$ or in regions with very diffuse as well as highly 


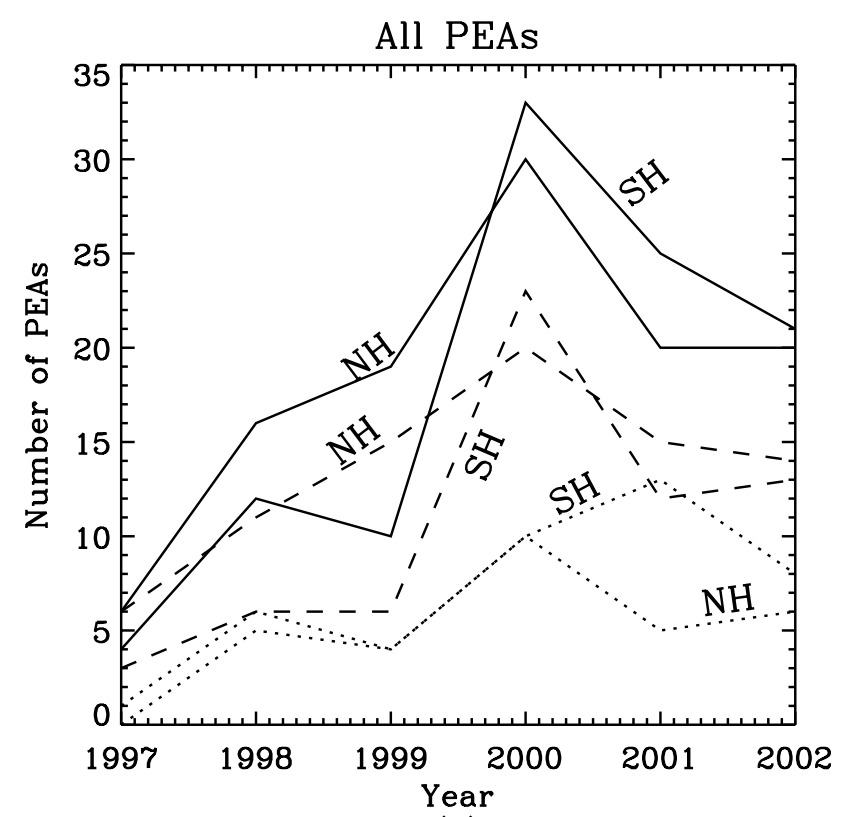

(a)

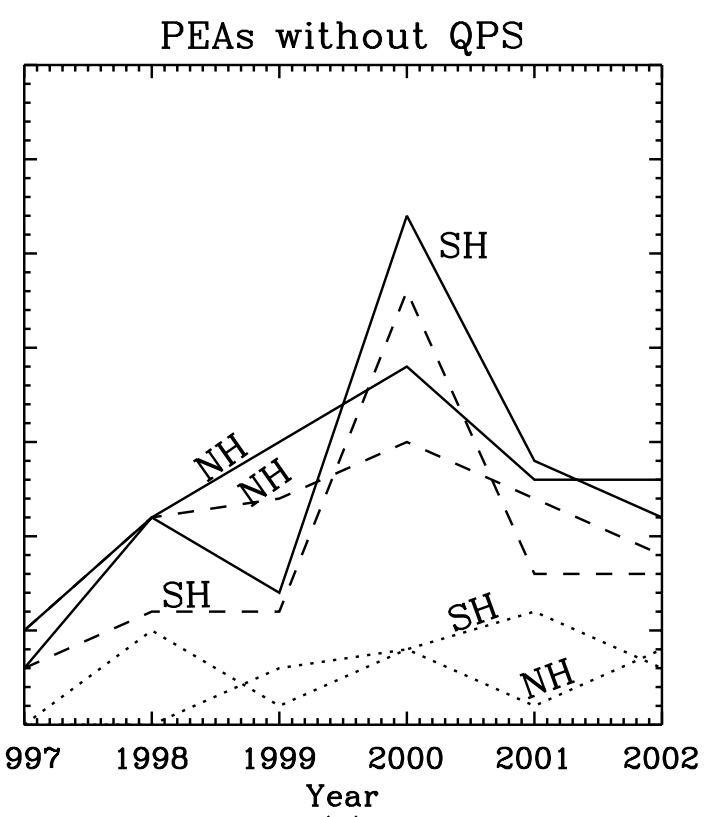

(b)

Fig. 17. a) Total number of PEAs identified in the Sun's northern and southern hemispheres (solid lines) during 1997-2002 based on SOHO/EIT $195 \AA$ A observations displayed together with the number of events with positive and negative magnetic polarities (dashed lines) to the West of the neutral line in northern and southern hemisphere respectively. The dotted lines represent the number of events with reversed polarities in both hemispheres. b) Same notation as in a), but with PEAs exhibiting quadrupolar-like (PEAs that formed in between pairs of BPRs) photospheric field source regions removed. Note that no events were recorded by EIT during the SOHO recovery phase in the second half of 1998.

complex photospheric fields that made the field associations doubtful. In some cases MDI data was not available. The spatial distribution of the 216 PEAs in heliographic coordinates and their magnetic polarities between 1997 and 2002 are displayed as yearly Carrington maps in Fig. 16. The color of each point at the lower latitude end of each PEA axis represents the magnetic polarity to the West of the polarity inversion line in the sense of solar rotation, with the red color labeling positive $(\mathrm{N})$ magnetic polarity and the blue color labeling negative $(\mathrm{S})$ magnetic polarity.

Figure 16 shows that the number of PEA events increases until solar maximum in 2000 and then remains at high level in 2001 and 2002, resembling the frequency of the yearly number of CMEs detected by LASCO (Gopalswamy et al. 2003). However, it should be noted that high latitude PEAs are probably lacking because of the selection criteria which require the PEA to be observable over its full spatial extent. The clustering of PEAs in some years at specific CR longitudes (e.g., near 250 degrees in 2000) may be compared with the clustering of active regions in the rising to maximum phase of solar cycle 23 pointed out by Pojoga \& Cudnik (2002) and the appearance of longitudinal bands of active regions reported by Benevolenskaya et al. (1999).

The yearly variation of the respective magnetic polarities found West of the neutral lines (PILs) in each solar hemisphere during 1997-2002 is presented in Fig. 17. In analogy to Fig. 16, the magnetic polarities to the West of the PILs are indicated with red and blue colors. As noted earlier, the expected sign of the leading magnetic polarities of BPRs is expected to be positive (North) in the northern hemisphere and vice versa in the southern hemisphere (compare with Figs. 14a,b). Out of the 216 events in Fig. 17a, 111 events were seen in the North (solid black line) and 105 in the South (dashed black line), i.e. the distribution was about equal in both hemispheres. The maximum number of PEAs peaks in both hemispheres at times of solar maximum in 2000. In the northern hemisphere 81 (73\%) out of 111 PEAs and in the southern hemispheres $63(60 \%)$ out of 105 matched the expected magnetic polarity (dashed lines in Fig. 17a). However, a fraction of the events showed reversed polarities (dotted lines). The PEAs that had formed in between pairs of BPRs, yielding reversed polarities in the given cycle, have not been taken into account. These cases do not contradict Hale's law, and have been subtracted in the numbers provided in Fig. 17b. The refined consideration reveals, that the expected polarity dominance for cycle 23 is getting more close to the total number of events, but still with a minority of reversed cases in both hemispheres. A hemispheric asymmetry in the distribution of evolving photospheric magnetic flux, as supported by the results of Li et al. (2002) who found more sunspot groups appearing in the northern hemisphere of the Sun in the rising to maximum phase of cycle 23, from 1996 until 2000, is not apparent in the PEA frequency distribution.

In order to investigate the solar cycle variation of the PEAs heliographic locations, the variation of the midpoints of the PEA axes during 1997-2002 were investigated. Figure 18 shows, that the PEAs locations follow, as expected, the butterfly pattern of sunspots. Similar to active regions, the PEAs move in latitude from shortly before solar minimum until sometime shortly after the following minimum from the higher latitudes towards the solar equator. Some PEA events at higher latitudes seem to coincide with periods when strong pulses of new magnetic flux caused a migration of the following polarity fields in BPRs towards the Sun's poles in agreement with the findings of Benevolenskaya et al. (2002). A linear polynomial fit 


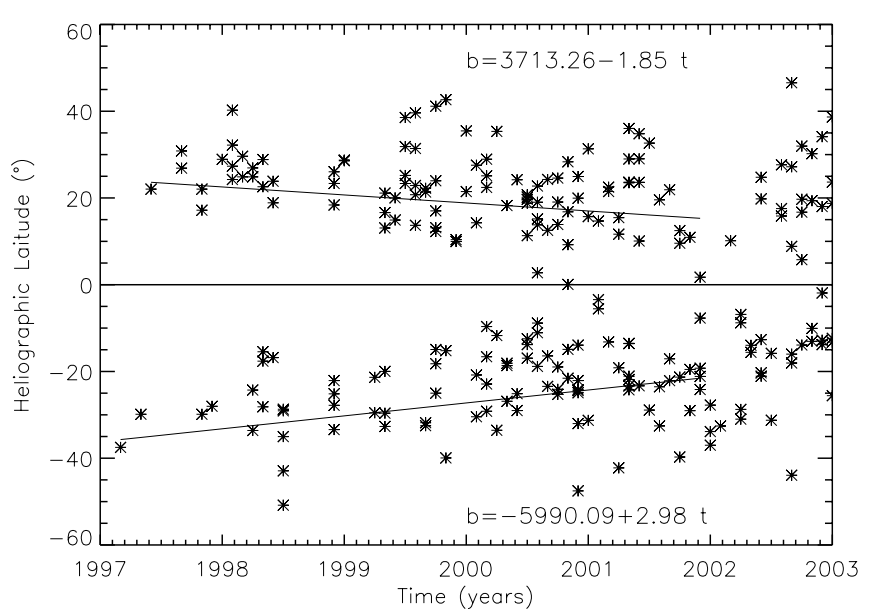

Fig. 18. Variation of the heliographic latitude of post-eruptive arcades in solar cycle 23 from 1997 to 2002 . The straight line represents fits for the Sun's northern (positive latitudes) and southern (negative latitudes) hemisphere. Note that no events were detected above $60^{\circ}$ latitude.

yielded for the latitude variation of the PEA locations in the two hemispheres: $b_{\mathrm{N}}(\mathrm{deg})=3713.26-1.85 t$ for the North and $b_{\mathrm{S}}(\mathrm{deg})=-5990.09+2.98 t$ for the South, with the time $\mathrm{t}$ given in years from 1997-2002. The source regions of the PEAs on average occurred at higher latitudes in the Sun's southern hemisphere compared to those in the northern hemisphere. The difference between $1997-2000$ is about $15^{\circ}$ in latitude. This effect may have caused the more frequent appearance of reversed polarity PEAs in the southern hemisphere.

\section{Summary and conclusions}

From a careful inspection of the full set of SOHO/EIT $195 \AA$ observations taken during the period 1997-2002, a set comprising 236 post-eruptive arcade (PEA) events has been selected, with the requirement that each PEA needed to be observable over its full spatial extent on the visible solar disk. The selected PEA events were correlated with SOHO/LASCO observations to investigate the association of PEAs with whitelight CMEs and their photospheric source regions were located in the SOHO/MDI synoptic charts.

The derived basic characteristics of EUV PEAs over the years 1997-2002 can be summarized as follows:

1. A near one to one correspondence between PEAs observed by SOHO/EIT at $195 \AA$ and white-light CMEs detected by $\mathrm{SOHO} / \mathrm{LASCO}$ was found for the selected events. PEAs can thus be considered as reliable disk tracers of CMEs, i.e. EUV images taken at $195 \AA$ can provide information on CME events even without the availability of simultaneous coronagraph observations.

2. A systematic study of the chronological evolution of the EUV and X-ray signatures in four events shows, based on SOHO, Yohkoh and GOES 8 measurements, that the rising phases of the X-ray flares coincided with the acceleration and lift-off phases of the corresponding CMEs, followed by the peaks in flare intensities and subsequent coronal brightening visible in soft X-rays and at EUV wavelengths. PEAs thus represent a coronal signature for the aftermath of a CME in the course of its magnetic restructuring.

3. EUV PEAs had $195 \AA$ emission lifetimes in the range 2 to $20 \mathrm{~h}$, with an average lifetime of $7 \mathrm{~h}$, i.e. PEAs still existed at times when the corresponding CME already reached distances of a couple of solar radii from the Sun, depending on its individual speed.

4. The length of the long axis, $L$, of a PEA varied in the range 2 to 40 degrees, with an average value of 15 degrees.

5. The heliographic positions were found to match the locations of the active region belts in both hemispheres, with no events identified at latitudes higher than $60^{\circ} \mathrm{N}$ or S and with an extremely rare $(<1 \%)$ number of transequatorial cases.

6. The orientations of the axes of the PEAs followed Joy's law, being commonly NE to SW oriented in the Sun's northern hemisphere and SE to NW in the southern hemisphere.

7. The lengths of the PEA axes increased with latitude by a factor of 3 to 4 in each hemisphere. The exact latitudinal variation during the period 1997 to 2002 was: $L_{\mathrm{N}}(\mathrm{deg})=2.9(b-17.1)$ in the northern and $L_{\mathrm{S}}(\mathrm{deg})=$ $-1.6(b+13.4)$ in southern hemisphere respectively, with $b$ being the heliographic latitude.

8. The PEAs spanned polarity inversion lines (PIL) separating regions of opposite magnetic polarities in the SOHO/MDI synoptic charts of the photospheric magnetic field. 149 (68\%) out of 216 PEAs, for which MDI data were available, formed in individual bipolar regions (BPRs), the remaining ones originated from neighboring pairs of BPRs.

9. The dominant polarity found to the West of the PEA axes overlying the polarity inversion lines separating the opposite field polarities, was as expected predominantly positive $(\mathrm{N})$ in the northern hemisphere and predominantly negative (S) in the southern hemisphere during 1997-2002. However, a significant number (31\% out of 216 events) showed the reversed polarity being randomly distributed over the investigated time period.

10. The heliographic latitude variation of the PEA positions at the Sun followed the active region locations during 1997-2002, with $b_{\mathrm{N}}(\mathrm{deg})=3713.26-1.85 t$ in the North and $b_{\mathrm{S}}(\mathrm{deg})=-5990.09+2.98 t$ in the South, where $t$ is given in years from 1997 to 2002. During the analyzed time interval, PEAs in the southern hemisphere were found on average to be located about $15^{\circ}$ further away from the solar equator compared to the position of PEAs in the northern hemisphere.

It is interesting to note, that for some of the PEA events of this study the corresponding CMEs caused major geomagnetic storms, like the CME released in association with the giant PEA on July 14, 2000 or the minor storm caused by the CME associated with the PEA on February 17, 2000 (e.g., Bothmer 2003; Yurchyshyn et al. 2001). In both cases, the in situ magnetic field properties of the CMEs matched the ones predicted from the photospheric field patterns of the CMEs' source regions according to the scenario presented by Bothmer \& Schwenn (1994, 1998) and Bothmer \& Rust (1997). A systematic study addressing the question which of the CMEs associated with the PEAs selected in this study did cause 
geomagnetic storms and whether the in situ field structure of the CMEs agreed in general with the expected source region field structures according to the Bothmer \& Schwenn (1994, 1998) scenario was beyond the scope of this study but will be important to investigate in further studies.

Beyond the potential of PEAs as disk signatures for improved space weather predictions, they also serve as important source region tracers of CMEs. The PEAs selected in this study provide a unique data set to study the underlying photospheric field configuration and possible onset mechanisms of CMEs. These aspects are of special interest for the interpretation of data provided by the NASA Solar-B and STEREO missions, with planned launches in 2005 and 2006.

Acknowledgements. The usefull comments and suggestions provided by Dr. K. P. Dere have greatly improved the quality of the manuscript. We are grateful to Prof. Dr. Rainer Schwenn and Prof. Dr. Sami K. Solanki for valuable discussions and comments. This study is part of the scientific investigations of the project Stereo/Corona supported by the German "Bundesministerium für Bildung und Forschung" through the "Deutsche Zentrum für Luft- und Raumfahrt e.V." (DLR, German Space Agency) under project number 50 OC 0005 . Stereo/Corona is a science and hardware contribution to the optical imaging package SECCHI, currently being developed for the NASA STEREO mission to be launched in 2005. We like to thank the SOHO/LASCO/EIT/MDI consortium for providing the data and the software libraries and we acknowledge use of the CME catalog generated and maintained by NASA and The Catholic University of America in cooperation with the Naval Research Laboratory. SOHO is a project of international cooperation between ESA and NASA. We acknowledge the use of data from the American-Japanese satellite Yohkoh provided by the MSSL SURF group (http://surfwww.mssl.ucl.ac.uk/surf) and of $\mathrm{H} \alpha$ data from the Observatory at Paris/Meudon distributed via the French BASS2000 (http://mesola.obspm.fr/present_en.html). The TRACE image is courtesy of http://vestige. lmsal. com/TRACE. TRACE is a mission of the Stanford-Lockheed Institute for Space Research (a joint program of the Lockheed-Martin Advanced Technology Center's Solar and Astrophysics Laboratory and Stanford's Solar Observatories Group), and part of the NASA Small Explorer program.

\section{References}

Benevolenskaya, E. E., Hoeksema, J. T., Kosovichev, A. G., \& Scherrer, P. H. 1999, ApJ, 517, L163

Benevolenskaya, E. E., Kosovichev, A. G., Lemen, J. R., et al. 2002, ApJ, 571, L181

Bothmer, V. 1999, in Proc. of ESA Workshop on Space Weather 1998 (Noordwijk: ESA), 117

Bothmer, V., \& Schwenn, R. 1994, Space Sci. Rev., 70, 215

Bothmer, V., \& Schwenn, R. 1995, J. Geomagn. \& Geoelectr., 47, 1127

Bothmer, V., \& Schwenn, R. 1998, Ann. Geophy., 16, 1

Bothmer, V., \& Rust, D. M. 1997, The Field Configuration of Magnetic Clouds and the Solar Cycle, in Coronal Mass Ejections, ed. N. Crooker, J. A. Joselyn, \& J. Feynman (Washington, DC: AGU), 139
Bothmer, V. 2003, in Proc. ISCS 2003 Symp., Solar Variability as an Input to the Earth's Enviornment, ed. A. Wilson (Noordwijk: ESA), 419

Brueckner, G. E., Howard, R. A., Koomen, M. J., et al. 1995, Sol. Phys., 162, 357

Brueckner, G. E., Delboudinière, J.-P., Howard, R. A., et al. 1998, Geophys. Res. Lett., 25, 3019

Canfield, R. C., Hudson, H. S., \& McKenzie, D. E. 1999, Geophys. Res. Lett., 26, 627

Delaboudinière, J.-P., Artzner, G. E., Brunaud, J., et al. 1995, Sol. Phys., 162, 291

Dere, K. P., Moses, J. D., Delaboudinière, J.-P., et al. 2000, Sol. Phys., 195,13

Gosling, J. T., Hildner, E., MacQueen, R. M., et al. 1974, J. Geophys. Res., 79, 4581

Gopalswamy, N., Alejandro, L., Yashiro, S., Nunes, S., \& Howard, R. A. 2003, in Proc. ISCS 2003 Symp., Solar Variability as an Input to the Earth's Enviornment, ed. A. Wilson (Noordwijk: ESA), 403

Hale, G. E., Ellerman, F., Nicholson, S. B., \& Joy, A. H. 1919, ApJ, 49, 153

Howard, R. A., Michels, D. J., Sheeley, N. R. Jr., \& Koomen, M. J. 1982, ApJ, 263, L101

Howard, R. A., Brueckner, G. E., St. Cyr, O. C., et al. 1997, Observations of CMEs from SOHO/LASCO, in Coronal Mass Ejections, ed. N. Crooker, J. A. Joselyn, \& J. Feynman (Washington, DC: AGU), 17

Hudson, H. S., Lemen, J. R., St. Cyr, O. C., Sterling, A. C., \& Webb, D. F. 1998, Geophys. Res. Lett., 25, 2481

Kopp, R. A., \& Pneuman, G. W. 1976, Sol. Phys., 50, 85

Li, Ke-Jun, Xiao-Hua, Yun, Hong-Sik, et al. 2002, Astron. Soc. Jpn, 54,629

Plunkett, S. P., Thompson, B. J., Howard, R. A., et al. 1998, Geophys. Res. Lett., 25, 2477

Pojoga, S., \& Cudnik, B. 2002, Sol. Phys., 208, 17

Rust, D. M., \& Webb, D. F. 1977, Sol. Phys., 54, 403

Scherrer, P. H., Bogart, R. S., Bush, R. I., et al. 1995, Sol. Phys., 162, 129

Sheeley, N. R. Jr., Howard, R. A., Koomen, M. J., et al. 1982, Space Sci. Rev., 33, 219

St. Cyr, O. C., Howard, R. A., Sheeley, N. R. Jr., et al. 2000, J. Geophys. Res., 105, 18169

Sterling, A. C., Hudson, H. S., Thompson, B. J., \& Zarro, D. M. 2000, ApJ, 532, 628

Subramanian, P., \& Dere, K. P. 2001, ApJ, 561, 372

Svestka, Z. K., Fárník, F., Hudson, H. S., \& Hick, P. 1998, Sol. Phys., 182,179

Tandenberg-Hanssen, E. 1974, Solar Prominences, 12 (DordrechtHolland/Boston-USA: D. Reidel Publishing Company)

Tsuneta, S., Action, L., Bruner, M., et al. 1991, Sol. Phys., 136, 37

Webb, D. F., \& Hundhausen, A. J. 1987, Sol. Phys., 108, 383

Webb, D. F. 2000, IEEE Trans. Plasma Physics, 28, 1795

Yashiro, S., Gopalswamy, N., Michalek, G., et al. 2002, AGU, SMSH32A

Yurchyshyn, V. B., Wang, H., Goode, P. R., \& Deng, Y. 2001, ApJ, 563,381

Zhang, J., Dere, K. P., Howard, R. A., et al. 2003, ApJ, 520

Zhang, J., Dere, K. P., Howard, R. A., Kundu, M. R., \& White, S. M. 2001, ApJ, 559, 452 